\title{
Numerical Solution of Compressible Steady Flows around the RAE 2822 Airfoil
}

\author{
P. Kryštůfek ${ }^{1, \text { a }}$, K. Kozel ${ }^{2}$ \\ ${ }^{1}$ Technical University of Liberec, Faculty of Mechanical Engineering, Czech Republic \\ ${ }^{2}$ Czech Technical University in Prague, Faculty of Mechanical Engineering, Czech Republic
}

\begin{abstract}
The article presents results of a numerical solution of subsonic, transonic and supersonic flows described by the system of Navier-Stokes equations in 2D laminar compressible flows around the RAE 2822 airfoil. Authors used FVM multistage Runge-Kutta method to numerically solve the flows around the RAE 2822 airfoil.
\end{abstract}

\section{Introduction}

The article follows the previous successful results [1] and contains a numerical code for simulating subsonic, transonic and supersonic flows field around the RAE 2822 airfoil. In these simulations the meshes type C has been used, which was created by mesh generator and has been described in [2-3]. In this case mesh has been created for numerical solution over profile RAE 2822.

\section{Mathematical models}

The 2D flow of a viscid compressible fluid (the Newtonian fluid) is described by the system of NavierStokes equations

$$
\mathbf{W}_{t}+\mathbf{F}_{x}+\mathbf{G}_{y}=\mathbf{R}_{x}+\mathbf{S}_{y},
$$

where

$$
\begin{aligned}
& \mathbf{W}=\left(\rho, \rho w_{1}, \rho w_{2}, e\right)^{T}, \\
& \mathbf{F}=\left(\rho w_{1}, \rho w_{1}^{2}+p, \rho w_{1} w_{2},(e+p) w_{1}\right)^{T}, \\
& \mathbf{G}=\left(\rho w_{2}, \rho w_{1} w_{2}, \rho w_{2}^{2}+p,(e+p) w_{2}\right)^{T}, \\
& \mathbf{R}=\left(0, \tau_{x x}, \tau_{x y}, w_{1} \tau_{x x}+w_{2} \tau_{x y}-q_{x}\right)^{T}, \\
& \mathbf{S}=\left(0, \tau_{x y}, \tau_{y y}, w_{1} \tau_{x y}+w_{2} \tau_{y y}-q_{y}\right)^{T},
\end{aligned}
$$

and [4]

$$
\begin{aligned}
p & =(\kappa-1)\left[e-\frac{1}{2}\left(w_{1}^{2}+w_{2}^{2}\right)\right], \\
\tau_{i j} & =\mu\left(\frac{\partial w_{i}}{\partial x_{j}}+\frac{\partial w_{j}}{\partial x_{i}}\right)-\frac{1}{3} \delta_{i j} \frac{\partial w_{k}}{\partial x_{k}}, \\
q_{j} & =-\frac{\kappa}{\kappa-1} \frac{\mu}{\operatorname{Pr}} \frac{\partial}{\partial x_{j}}\left(\frac{p}{\rho}\right) .
\end{aligned}
$$

In the above equations, $\mathbf{W}$ is conservative variable, $\mathbf{F}, \mathbf{G}$ are function of inviscid physical fluxes, $\mathbf{R}, \mathbf{S}$ are function of viscid physical fluxes, $\rho$ denotes density, $w_{1}, w_{2}$ are components of velocity in the direction of axis $x, y ; p$ is pressure, $e$ is total energy per unit volume. The parameter $\kappa=1,4$ is the adiabatic exponent. $\tau_{i j}$ represents shear stress, $q_{j}$ is heat flux (given by Fourier's Law assuming Mayer's formula), $\mu$ represents dynamical viscosity (calculated by Sutherland formula [4]) and $\operatorname{Pr}$ is laminar Prandtl number.

All variables were considered dimensionless and inflow variables (with the suffix $\infty$ ) were used as the reference variable for the transformation of the equations to dimensionless form. Thus after the transformation the system of Navier-Stokes equations is written

$$
\mathbf{W}_{t}+\mathbf{F}_{x}+\mathbf{G}_{y}=\frac{1}{\operatorname{Re}_{\infty}}\left(\mathbf{R}_{x}+\mathbf{S}_{y}\right)
$$

where $R e_{\infty}$ is a Reynolds number. 


\section{Numerical methods}

For modeling of the mentioned flow case, numerical scheme of FVM multistage Runge-Kutta (RK) is used on non-orthogonal structured grids of quadrilateral cells $D_{i j}$.

$$
\begin{aligned}
\mathbf{W}_{i, j}^{(0)} & =\mathbf{W}_{i, j}^{(n)}, \\
\mathbf{W}_{i, j}^{(r+1)} & =\mathbf{W}_{i, j}^{(0)}-\Delta t \cdot \alpha_{r} \cdot \operatorname{Rez} \mathbf{W}_{i, j}^{(r)}+A D\left(\mathbf{W}_{i, j}^{(r)}\right), \\
\mathbf{W}_{i, j}^{(n+1)} & =\mathbf{W}_{i, j}^{(m)},
\end{aligned}
$$

$\operatorname{Rez}_{i, j}^{(n)}=\frac{1}{\left|D_{i, j}\right|} \sum_{k=1}^{4}\left[\left(\tilde{\mathbf{F}}_{k}^{n}-\frac{1}{\operatorname{Re}} \tilde{\mathbf{R}}_{k}^{n}\right) \Delta y_{k}-\left(\tilde{\mathbf{G}}_{k}^{n}-\frac{1}{\operatorname{Re}} \tilde{\mathbf{S}}_{k}^{n}\right) \Delta x_{k}\right]$

for

$$
\begin{gathered}
r=0,1, \ldots, m-1 ; m=5 \\
\alpha_{1}=0,25 ; \alpha_{2}=0,1667 ; \alpha_{3}=0,375 ; \alpha_{4}=0,5 ; \alpha_{5}=1
\end{gathered}
$$

where $\left|D_{i, j}\right|$ represents the surface or volume of the cell. Numerical approximations $\tilde{\mathbf{F}}, \tilde{\mathbf{G}}$ of convective terms $\mathbf{F}, \mathbf{G}$ were considered in the forward form of the first order of accuracy. Numerical approximations $\tilde{\mathbf{R}}, \tilde{\mathbf{S}}$ of dissipative terms $\mathbf{R}, \mathbf{S}$ were approximated in the central form of the second order of accuracy and by using dual cells and applying Green's formula.

The scheme was extended to include Jameson's artificial dissipation in (5) because of the stability of the method

$$
\begin{aligned}
A D\left(\mathbf{W}_{i, j}^{(n)}\right)= & k_{1} \cdot \gamma_{i} \cdot\left(\mathbf{W}_{i+1, j}^{(n)}-2 \mathbf{W}_{i, j}^{(n)}+\mathbf{W}_{i-1, j}^{(n)}\right) \\
& +k_{2} \cdot \gamma_{j} \cdot\left(\mathbf{W}_{i, j+1}^{(n)}-2 \mathbf{W}_{i, j}^{(n)}+\mathbf{W}_{i, j-1}^{(n)}\right),
\end{aligned}
$$

where

$$
\begin{aligned}
& \gamma_{i}=\frac{\left|p_{i+1, j}^{n}-2 p_{i, j}^{n}+p_{i-1, j}^{n}\right|}{\left|p_{i+1, j}^{n}\right|+2\left|p_{i, j}^{n}\right|+\left|p_{i-1, j}^{n}\right|}, \\
& \gamma_{j}=\frac{\left|p_{i, j+1}^{n}-2 p_{i, j}^{n}+p_{i, j-1}^{n}\right|}{\left|p_{i, j+1}^{n}\right|+2\left|p_{i, j}^{n}\right|+\left|p_{i, j-1}^{n}\right|} .
\end{aligned}
$$

The convergence to the steady state is followed by log L2 residual defined by

$$
\operatorname{Rez}_{i, j}^{(n)}=\frac{1}{N} \sqrt{\sum_{i, j}\left(\frac{\mathbf{W}_{i, j}^{(n+1)}-\mathbf{W}_{i, j}^{(n)}}{\Delta t}\right)^{2}}
$$

where $\mathrm{N}$ is a number of all elements in the computational domain.

\section{Specification of test case}

We used RAE 2822 airfoil. This airfoil was presented in figure 1 .

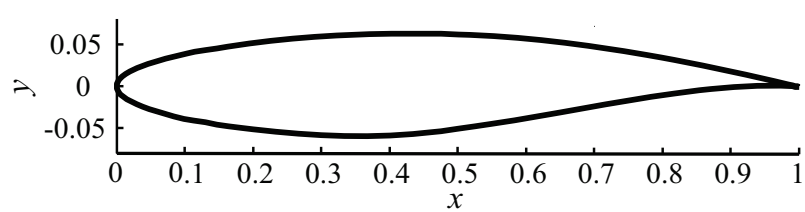

Figure 1. RAE 2822 airfoil (by Kryštůfek).

The outlines of the computational domain are shown in figure 2. In this computational domain was placed RAE 2822 airfoil.

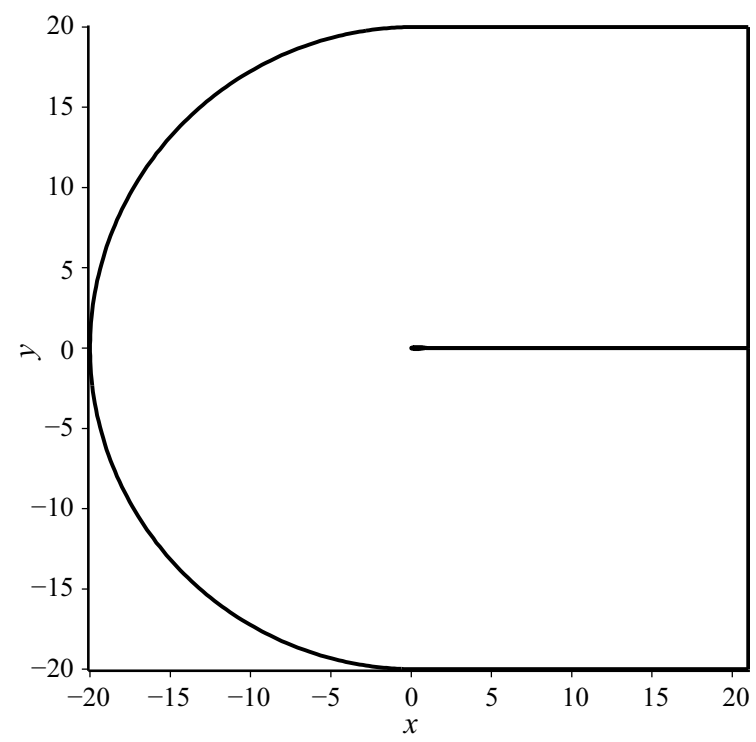

Figure 2. Computational domain.

We selected for numerical solution a structured mesh formed by quadrilateral finite volumes. The grid around profiles (wing) usually consists of a $\mathrm{C}$-grid in the flow direction. In the case of the C-topology the aerodynamics body is enclosed by one family grid lines, which also form the wake region.

The initial grid is generated algebraically by using the linear TFI method [5]. Afterwards, elliptic PDE's are employed to produce boundary-orthogonal grid with specific wall spacing. The RAE 2822 airfoil contour is approximated by a Bezier spline [6].

The grid with $384 \times 64$ elements created by ours program for this simulation were presented in figure 3 . The detail of grid near wall was presented in figure 4 .

\subsection{Boundary conditions}

For supersonic inflow the conservative variables on the boundary are determined by freestream values only. The values are specified based on the given Mach number $M a_{\infty}$ and two flow angles (angle of attack and side-slip angle). 


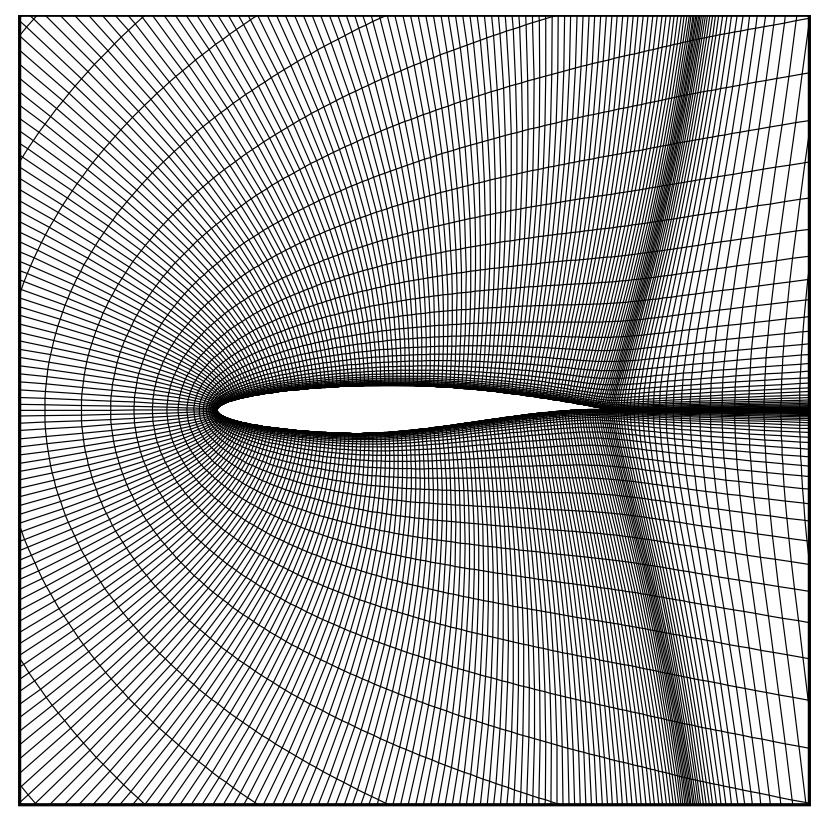

Figure 3. Viscid, C-type, RAE 2822 airfoil, 384x64 elements (by Kryštůfek [1]).

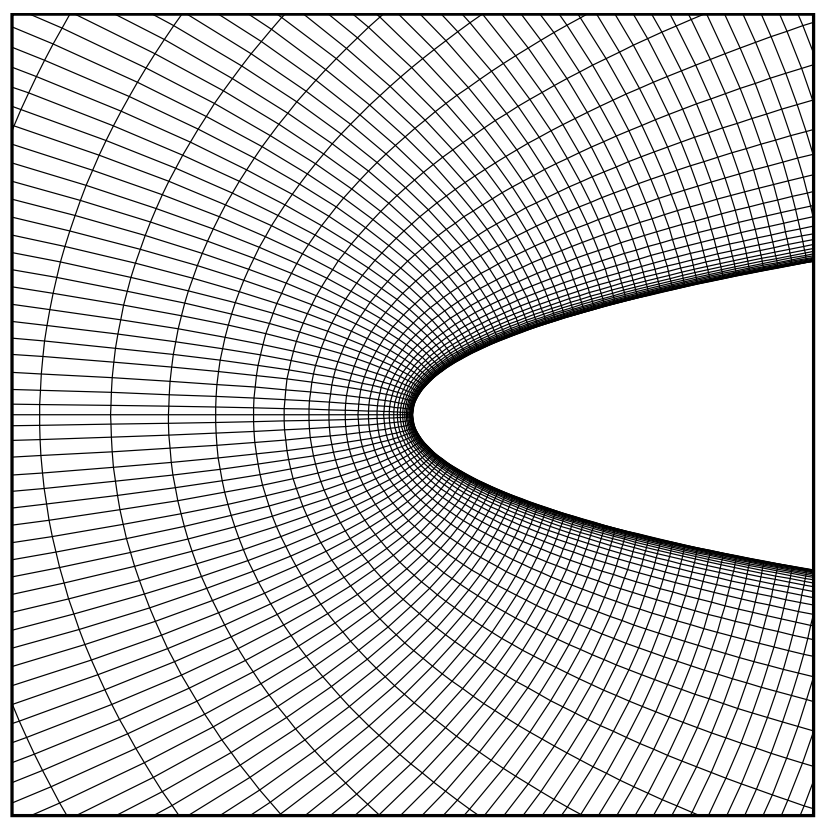

Figure 4. The detail of grid near wall, C-type, RAE 2822 airfoil, 384x64 elements (by Kryštůfek).

For supersonic outflow all conservative variables at the boundary must be determined from the solution inside domain.

Three characteristic variables are prescribed based on the free stream values at far field subsonic inflow. One characteristic variable is extrapolated from interior of the physical domain.

In the case of subsonic outflow, three flow variables (density and the two velocity components) have to be extrapolated from interior of the physical domain. The remaining four variables (pressure) must be specified externally (as a multiple of the input pressure).

On wall zero derivatives of velocity vector along normal is considered. This condition is implemented by using virtual cells. Such cells adjoin from outside on the boundary cells and we prescribe values of unknowns inside of them to obtain the desired effect.

\section{Numerical results and conclusion}

As mentioned above, the authors have dealt with the numerical simulation of 2D laminar transonic flows around the RAE 2822 airfoil and applied the multistage Runge-Kutta method and orthogonal structured grids $\mathrm{C}$ type with 384x64 cells for its created by the linear TFI method [4]. The authors considered a few different values of inlet Mach number $M a_{\infty} \in(0.4,1.1)$, Reynolds numbers $R e_{\infty}=5 \times 10^{6}$ and angle of attack $\alpha=0^{\circ}$. The numerical results are presented in Mach number isolines. On figures 5, 7, 9 are shown Mach number isolines for laminar compressible flow around RAE 2822 airfoil.

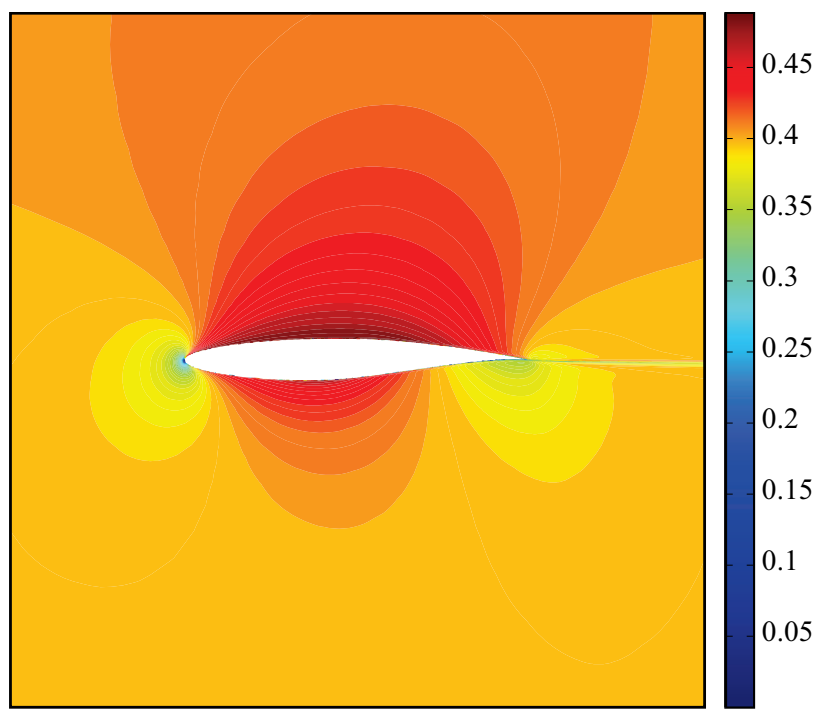

Figure 5. Laminar compressible flow around RAE 2822 airfoil, C-type mesh with $384 \times 64$ cells, Mach number isolines - RK scheme, $M a_{\infty}=0.4$ and $R e_{\infty}=5 \times 10^{6}, \alpha=0^{\circ}$.

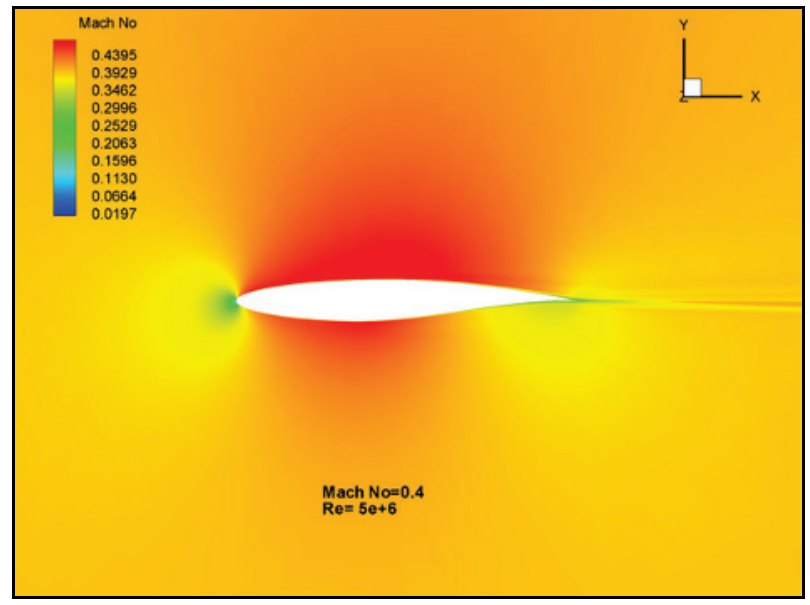

Figure 6. Laminar compressible flow around RAE 2822 airfoil, $M a_{\infty}=0.4$ and $R e_{\infty}=5 \times 10^{6}, \alpha=0^{\circ}$ by [7]. 


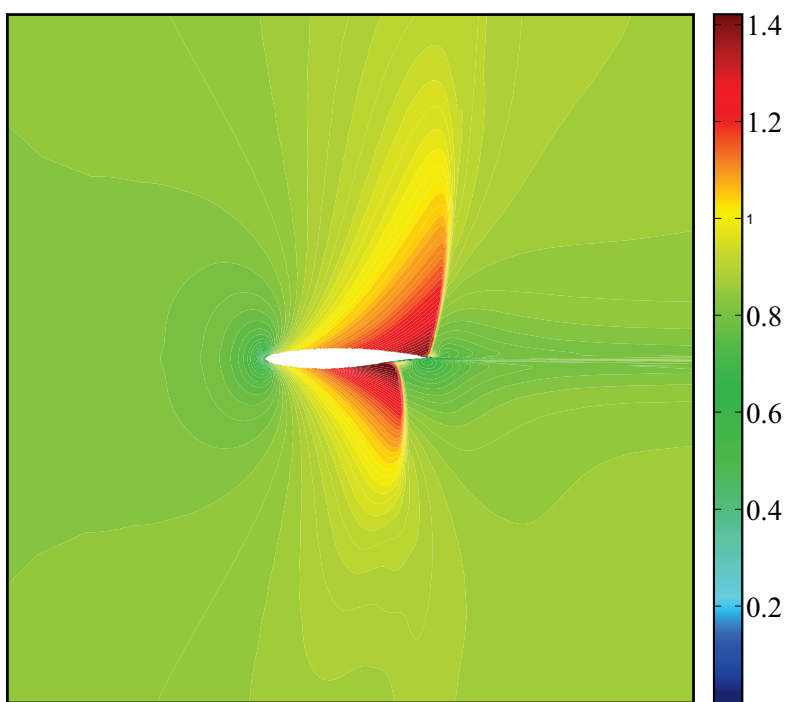

Figure 7. Laminar compressible flow around RAE 2822 airfoil, C-type mesch with 384x64 cells, Mach number isolines - RK scheme, $M a_{\infty}=0.85$ and $\operatorname{Re}_{\infty}=5 \times 10^{6}, \alpha=0^{\circ}$.

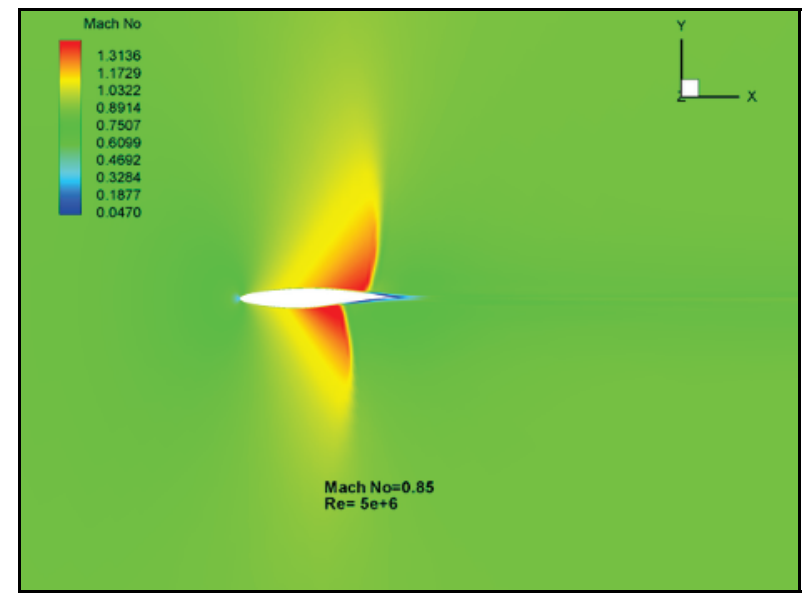

Figure 8. Laminar compressible flow around RAE 2822 airfoil, $M a_{\infty}=0.85$ and $R e_{\infty}=5 \times 10^{6}, \alpha=0^{\circ}$ by [7].

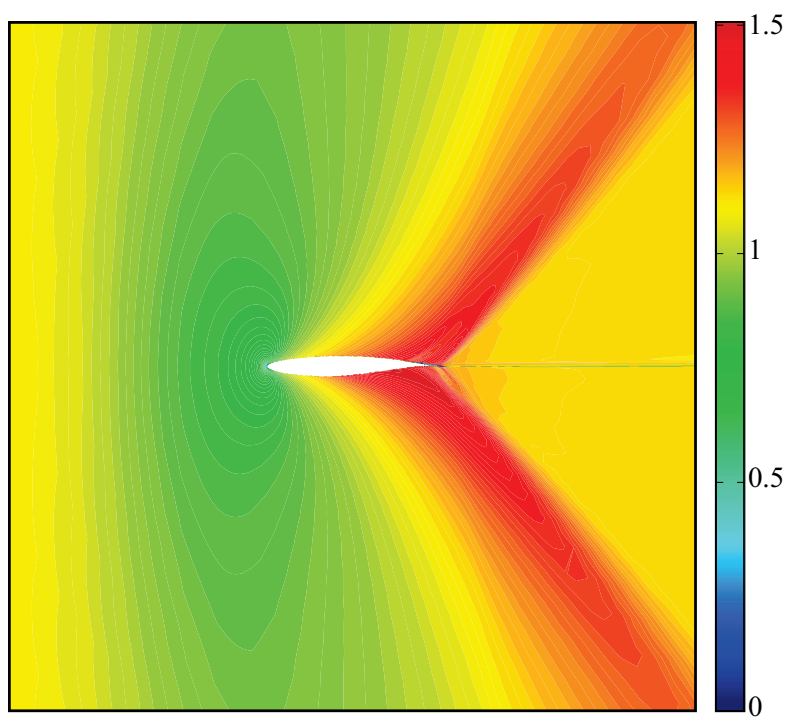

Figure 9. Laminar compressible flow around RAE 2822 airfoil, C-type mesh with 384x64 cells, Mach number isolines - RK scheme, $M a_{\infty}=1.1$ and $R e_{\infty}=5 \times 10^{6}, \alpha=0^{\circ}$.

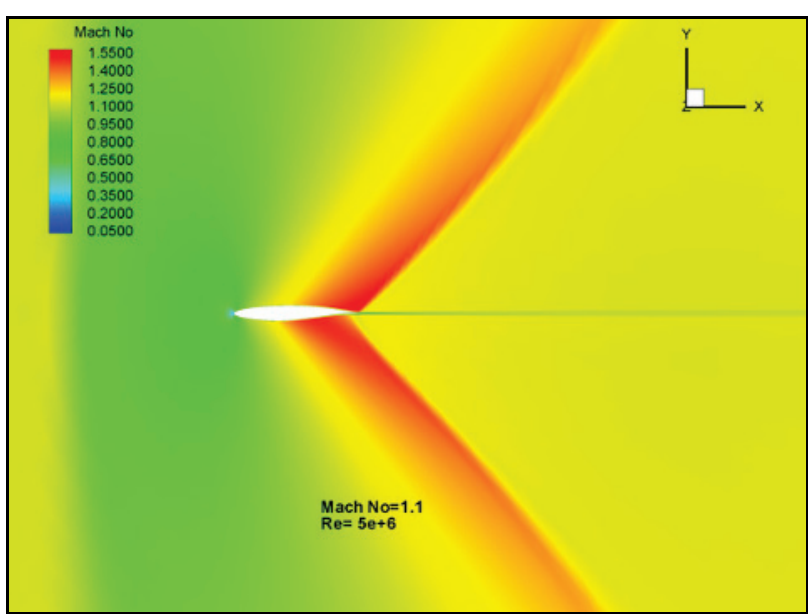

Figure 10. Laminar compressible flow around RAE 2822 airfoil, $M a_{\infty}=1.1$ and $R e_{\infty}=5 \times 10^{6}, \alpha=0^{\circ}$ by [7].

On figures 6, 8, 10 are shown Mach number isolines for same test case by Aerodynamic Design Simulation at The University of Sheffield [7]. The results are in good agreement. Shock wave is shifted further along the profile for case of transonic flow.

The results can be improved by suitable choice of parameters of Jameson's artificial dissipation. In the future, we will continue with an algebraic turbulence model.

\section{Acknowledgements}

This work was financially supported by the particular research student grant SGS 28000 at TU of Liberec.

\section{References}

1. P. Kryštůfek, K. Kozel, Experimental fluid mechanics, 371-374 (2012)

2. P. Kryštůfek, K. Kozel, Experimental fluid mechanics, 771-777 (2011)

3. P. Kryštůfek, K. Kozel, Power System Engineering, Thermodynamics \& Fluid Flow (2012)

4. J. Blazek, Computational Fluid Dynamics: Principles and Applications (2005)

5. A.J.W. Sommerfeld, Partial Differential Equations in Physics (1949)

6. F. M. White, Fluid Mechanics - Fourth Edition (2001)

7. Aerodynamic Design Simulation, TUOS, http://mec424.group.shef.ac.uk 